\title{
Mellin Transform-Based Correction Method for Linear Scale Inconsistency of Intrusion Events Identification in OFPS
}

\author{
Baocheng WANG ${ }^{1}$, Dandan $\mathrm{QU}^{2}$, Qing TIAN ${ }^{2 *}$, and Liping PANG ${ }^{3}$ \\ ${ }^{1}$ School of Computer, North China University of Technology, Beijing 100144, China \\ ${ }^{2}$ School of Electrical and Information Engineering, North China University of Technology, Beijing 100144, China \\ ${ }^{3}$ School of Aviation Science and Engineering, Beijing University of Aeronautics and Astronautics (BUAA), Beijing \\ 100191, China \\ "Corresponding author: Qing TIAN E-mail: hwm_hebust@163.com
}

\begin{abstract}
For the problem that the linear scale of intrusion signals in the optical fiber pre-warning system (OFPS) is inconsistent, this paper presents a method to correct the scale. Firstly, the intrusion signals are intercepted, and an aggregate of the segments with equal length is obtained. Then, the Mellin transform (MT) is applied to convert them into the same scale. The spectral characteristics are obtained by the Fourier transform. Finally, we adopt back-propagation (BP) neural network to identify intrusion types, which takes the spectral characteristics as input. We carried out the field experiments and collected the optical fiber intrusion signals which contain the picking signal, shoveling signal, and running signal. The experimental results show that the proposed algorithm can effectively improve the recognition accuracy of the intrusion signals.
\end{abstract}

Keywords: Linear scale; OFPS; MT; BP neural network; spectral characteristics

Citation: Baocheng WANG, Dandan QU, Qing TIAN, and Liping PANG, "Mellin Transform-Based Correction Method for Linear Scale Inconsistency of Intrusion Events Identification in OFPS,” Photonic Sensors, 2018, 8(3): 220-227.

\section{Introduction}

With the increasing demand of security in the fields of borders and oil pipelines, the optical fiber pre-warning system (OFPS) has attracted growing attention [1-3]. The optical fiber sensor has many advantages, such as anti-electromagnetic interference, good electrical insulation, passive sensors, high sensitivity, wide measuring range, and strong adaptability to various environmental conditions $[4,5]$. With these characteristics, it is widely used in the OFPS to realize the intrusion information collection. The OFPS exploits the laying of the underground cable to detect and identify the external intrusion signals so that it can obtain the place and time of the occurrence of the abnormal event. Extracting the effective features to improve the recognition performances is a key technique in identification [6].

In recent years, the methods for feature extraction of optical fiber intrusion signals are mainly studied from the views of the time and frequency domains. Reference [7] proposed the threshold-crossing rate algorithm to extract time domain characteristics of the collected signals. However, this method is ineffective for non-linear

Received: 29 December 2017 / Revised: 3 April 2018

(C) The Author(s) 2018. This article is published with open access at Springerlink.com

DOI: 10.1007/s13320-018-0486-9

Article type: Regular 
system. Reference [8] also proposed a robust method to identify the optical fiber intrusion, which extracted the time-domain characteristics of the signals as inputs into the neural network for training and recognition. However, the adaptive threshold is almost decided by experience without definite mathematical reasoning. References $[9,10]$ analyzed the intrusion signals from the frequency domain. Reference [11] proposed a 3-layer back-propagation artificial neural network (BP-ANN) to determine and identify the feature samples, which extracted the time-frequency domain characteristics of the signals as inputs. It was found that intrusions could be recognized clearly in a period less than one tenth of that by conventional dynamic time warping, and it has a merit of mitigating influence of environmental noises. After data processing, it was found by observation that there existed different scales among the collected signals, which posed a challenge for the extraction of characteristics effectively and further reduced the recognition rate.

The issue of scale exists not only in the OFPS, but also in many other areas such as image matching and radar target recognition. Conventional methods for matching image show well performance in solving the problem of image translation, but it is not correct for the case of rotation and scaling. To solve the problem, [12-14] proposed a matching algorithm based on the Fourier-Mellin transform, which can improve the recognition accuracy significantly. For radar target, there appears large scale inconsistence among the radar waveforms acquired in a scan cycle, which has an impact on radar target recognition. References [15-17] adopted the Mellin transform (MT) to eliminate this influence on recognition, which was caused by scale difference of the radar signal. The above literatures showed that the MT could solve the problem of scale inconsistency and improve the recognition rate. Inspired by its applications in these fields, the MT can be used to solve the scale transformation problem in the OFPS.
In this paper, the MT is applied to correct the scale of the optical fiber intrusion signal for the first time, and a recognition method based on the MT is proposed, which can be divided into two parts: scale correction and BP neural network. In the scale correction, we firstly pre-process the signals to obtain an aggregate of the segments with an equal length. Then, the MT is applied to convert them into the same scale. Herein, the signals with the consistent scale are obtained, which can guarantee the identification accuracy for the next step. Among the intrusion signals in each type, the randomness of the intrusion signals leads to the existence of signal shift transformation in the time domain, which has an impact on identification. In order to solve the problem, the Fourier transform is applied to obtain the spectral information while removing the delay effect. Finally, we take the spectral characteristics obtained by the Fourier transform as input to BP neural network for identification. Finally, we conduct a field experiment and use the proposed method to analyze the collected data in detail. The experimental results show that the algorithm can solve the problem of linear scale inconsistency and reduce the impact caused by time delay. Moreover, the signal recognition rate is obviously improved in the research of the intrusion signal identification of the OFPS.

The paper is organized as following. In Section 2, we introduce the scale correction method for signals based on the MT. The details of intrusion signal recognition algorithm are described in Section 3. The experimental analysis of the actual data is given in Section 4. Finally, the conclusion is provided in Section 5.

\section{Correction method for signal scale based on MT}

The flow chart of signal scale correction based on the MT is shown in Fig. 1. Firstly, the only template is selected from each specific type of intrusion signals, that is, one template corresponds 
to one type of data. Then, the template and signals are pre-processed to realize de-noising. Thirdly, the energy between the template and signals is compared to select the segment to be converted. Finally, the MT is applied for the selected signals to obtain the signals with the consistent scale.

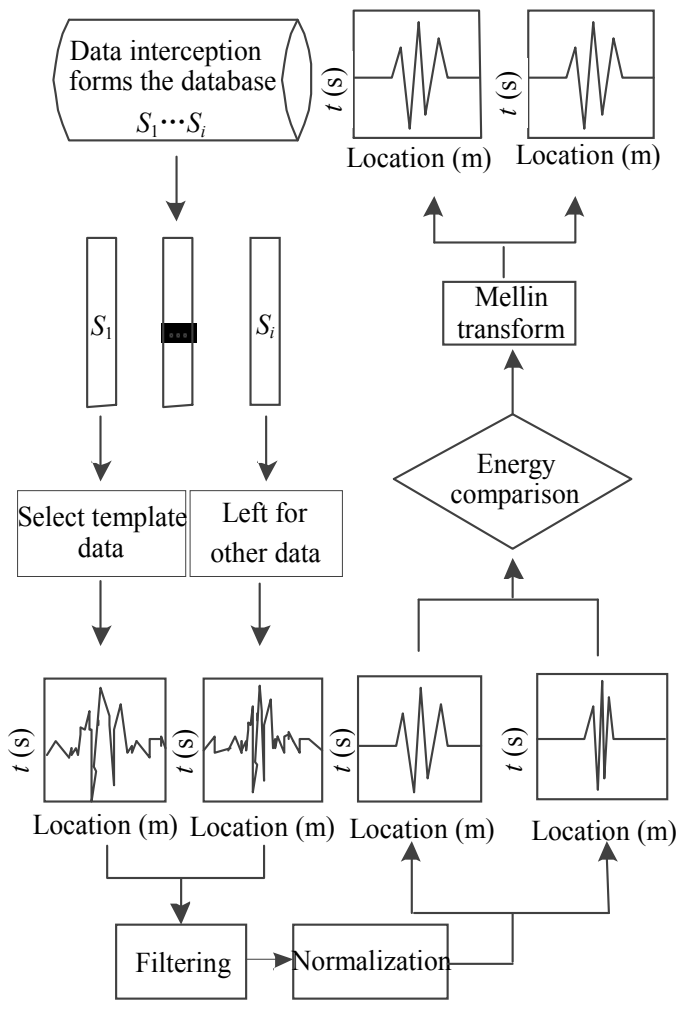

Fig. 1 Flow chart of signal scale correction based on the MT.

\subsection{Selection of the segments to be converted}

Firstly, the intrusion signals are intercepted, and an aggregate of the segments with an equal length which contains the whole intrusion information is obtained. The temporal resolution of each segment is $1 \mathrm{~ms}$. Moreover, the template is selected from the aggregate of segments to make all the segments with the same scale as the template. Then, band-pass filtering is performed on all intrusion signals for de-noising. We compare the energies of the signals and template to select the segments which need to be converted by setting the threshold. For the segment which meets the threshold, the scale correction method based on the MT is adopted to convert the scale of signals.

\subsection{Scale correlation method on intrusion signals}

The scaling correction method based on the MT is shown in Fig. 2. Assume that the initial collected optical fiber signals are $s_{1}(t)$ and $s_{2}(t)$, wherein $t$ presents time. There exists the scale factor between $s_{1}(t)$ and $s_{2}(t)$ which is $a$, and the relationship between two signals can be expressed as follows:

$$
s_{2}(t)=s_{1}(a t) .
$$

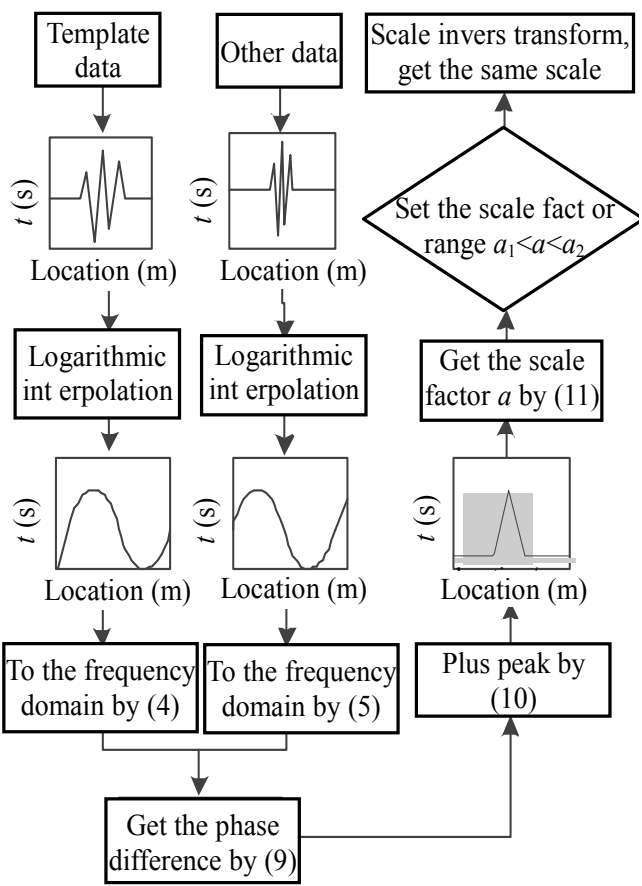

Fig. 2 Flow chart of the MT.

Firstly, $s_{1}(t)$ and $s_{2}(t)$ are performed logarithmic interpolation on the time axis, and the signals become $s_{1}(\lg t)$ and $s_{2}(\lg t)$, which are closed to discrete signals. Thus, the relationship between $s_{1}(t)$ and $s_{2}(t)$ in the logarithmic coordinate system is as follows:

$$
\begin{aligned}
s_{2}(\lg t) & =s_{1}(\lg a t) \\
& =s_{1}(\lg t+\lg a) .
\end{aligned}
$$

Equation (2) can be equivalent as

$$
s_{2}(x)=s_{1}(x+A)
$$

wherein

$$
x=\lg t
$$




$$
A=\lg a .
$$

Herein, the relationship between $s_{1}(t)$ and $s_{2}(t)$ is transformed into (3), which exists the translational amount, $A$, after the logarithmic interpolation. It indicates that the scaling can be solved by the translation invariance of the Fourier transform.

A classical technique for frequency analysis is the Fourier transform. The signals, $s_{1}(x)$ and $s_{2}(x)$, are presented in the frequency domain, which can be written as follows:

$$
\begin{gathered}
F_{1}(\omega)=\int_{-\infty}^{\infty} s_{1}(x) \mathrm{e}^{-\mathrm{j} \omega x} d x \\
F_{2}(\omega)=\int_{-\infty}^{\infty} s_{2}(x) \mathrm{e}^{-\mathrm{j} \omega x} d x \\
=\int_{-\infty}^{\infty} s_{1}(x+A) \mathrm{e}^{-\mathrm{j} \omega x} d x .
\end{gathered}
$$

There exists time shift characteristic in the Fourier transform, which can be expressed as

$$
\begin{aligned}
f(t) & \leftrightarrow F(\mathrm{j} \omega) \\
f\left(t \pm t_{0}\right) & \leftrightarrow F(\mathrm{j} \omega) \mathrm{e}^{ \pm \mathrm{j} \omega t_{0}}
\end{aligned}
$$

where $t_{0}$ is the translation amount. With this characteristic, $F_{1}(\omega)$ and $F_{2}(\omega)$ have the following relationship:

$$
F_{2}(\omega)=F_{1}(\omega) \mathrm{e}^{\mathrm{j} \omega A} .
$$

Equations (3) and (10) show that the translation in the time domain causes the phase changes of the signal in the frequency domain, which can be written as follows:

$$
\frac{F_{1}(\omega)}{F_{2}(\omega)}=\mathrm{e}^{-\mathrm{j} \omega A} .
$$

The Fourier inverse transformation of phase difference is applied to obtain a pulse function. The peak position of this function is the translation of $s_{1}(x)$ and $s_{2}(x), A$. The Fourier inverse transformation is as follows:

$$
\begin{aligned}
f(x) & =\frac{1}{2 \pi} \int_{-\infty}^{\infty} \mathrm{e}^{-\mathrm{j} \omega A} \mathrm{e}^{\mathrm{j} \omega x} d x \\
& =\delta(x-A) .
\end{aligned}
$$

Translation amount, $A$, is the scale factor between $s_{1}(t)$ and $s_{2}(t)$ in logarithmic coordinates, which can be restored to the scale factor in the rectangular coordinate:

$$
a=\mathrm{e}^{A} .
$$

Since there exists a slight difference of scale among the signals with the same type when being disturbed by outside interference, we define the threshold of the scale factor which is $a_{1}<a<a_{2}$ according to the priori information for selecting the signals that need to be converted. Finally, the signals are transformed according to the scale factor $a$, and the signal with the consistent scale is obtained by removing the effect on identification caused by the scale inconsistency.

\section{Spectral characteristics-based recognition algorithm for OFPS}

\subsection{Spectral characteristics extraction based on the Fourier transform}

Among the optical fiber intrusion signals in each type, the randomness of the intrusion signals leads to the existence of signal shift transformation in the time domain. So the initial time of the intrusion signal in each segment is different. The BP neural network is a classical algorithm for classification. In the BP neural network, the neurons between the layers realize an entire connection, that is to say each neuron of the next layer realizes weight connection with each neuron of the previous layer, and all neurons of each layer are not connected mutually. The learning process of the BP neural network is forward-propagating, namely providing the input information, and then forward propagating the input information is onto the hidden nodes of the hidden layer through the input layer. Finally, by operating the activation function of each cell, the output information of the hidden nodes is propagated to the nodes of the output layer, and then the actual output value of each cell is obtained. With these characteristics, the BP neural network cannot realize weight sharing, which causes some difficulty in identifying the signals with the scale inconsistency. Therefore, the Fourier transform is 
applied for signals to eliminate the effect of the translational transformation before classification.

Assume that the optical fiber intrusion signals, $s_{3}(t)$ and $s_{4}(t)$, which exist translational amount, $t_{1}$, can be expressed as follows:

$$
s_{3}(t)=s_{4}\left(t-t_{1}\right) \text {. }
$$

Herein, the spectrums of them are the same, as shown in the following equation:

$$
\left|F_{3}(\omega)\right|=\left|F_{4}(\omega)\right| .
$$

Therefore, features obtained by the Fourier transform have translational invariance, which can effectively solve the identification problems.

\subsection{Identification method of the BP neural network}

The BP neural network can be divided into three parts which are the input layer, hidden layer, and output layer. The BP network used in this paper, as shown in Fig. 3, includes 128-dimensional input, one hidden layer which contains 16 neurons, and 3-dimensional output. Sigmoid function is selected as non-linear excitation function of the hidden layer and the output layer node.

The spectrum characteristics obtained in Section 3.1 are sent into the BP neural network for training and classification.

128-dimensional Hidden layer 3-dimensional input (16 neurons) output

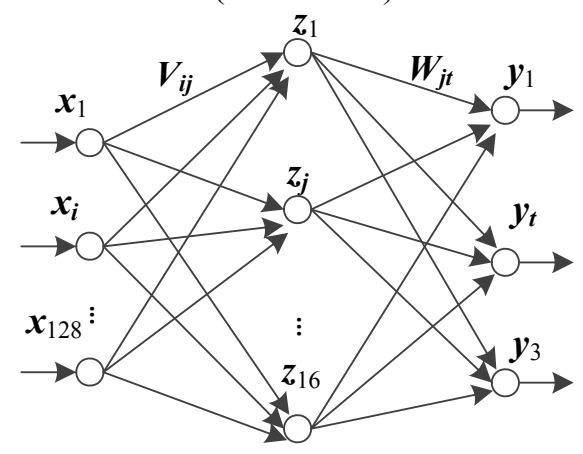

Fig. 3 BP neural network structure.

\section{Experimental simulation and results}

\subsection{Scale correction results and analysis}

We carried out the field experiments and collected the optical fiber intrusion signals which contain the picking signal, shoveling signal, and running signal. The intrusion signals are intercepted, and an aggregate of the segments with an equal length is obtained which contains the whole intrusion information. Firstly, it is verified whether there is scale transformation in the intrusion signal part of these data, then the MT is used to correct the scale of them, and the signals with the same scale are obtained.

The template is selected from the aggregate of signals, and then the MT is used for all of the signals. The scale scaling factor values between template signals are shown in Table 1.

Table 1 Scaling factor.

\begin{tabular}{ccc}
\hline & Intrusion type & $a$ \\
\hline Type 1 & Picking & 1.297 \\
Type 2 & Shoveling & 1.149 \\
Type 3 & Running & 1.193 \\
\hline
\end{tabular}

The waveform comparison between the selected template signals is shown in Fig. 4, where $L_{1}$ is the length of the template, and $L_{2}$ is the length of signals. It can be seen from Fig. 4 that the length of the intrusion signal for each type is not equal, which proves that the same type of signal exists scale inconsistency. We define the threshold of scale factor which is $0.5<a<2$ according to the priori information for selecting the signals that need to be converted. The above signals are processed by the scale correction method, and the MT is applied to convert the template and signals to the same scale. The results are shown in Fig. 5. After this, the template and signals have the same length, $L_{1}$. It illustrates that signals which belong to the same type can be effectively converted to the same scale by the scale correction method. The effect on the recognition of the optical fiber intrusion signal caused by the scale inconsistency is eliminated, and the translation invariant feature is obtained. 


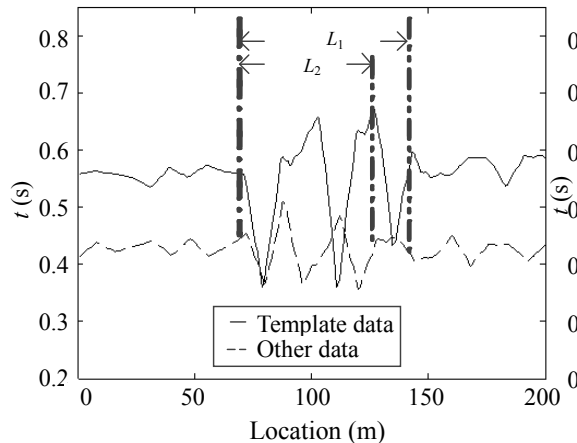

(a)

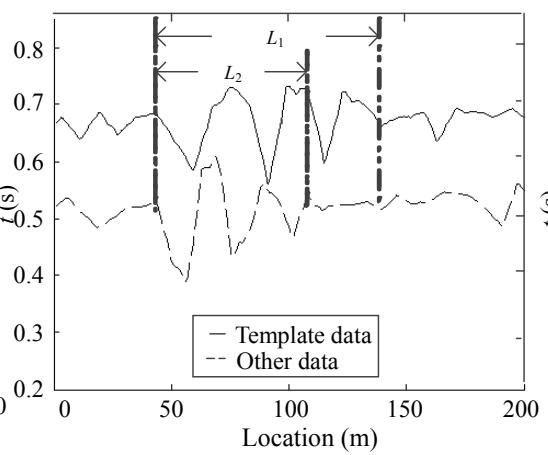

(b)

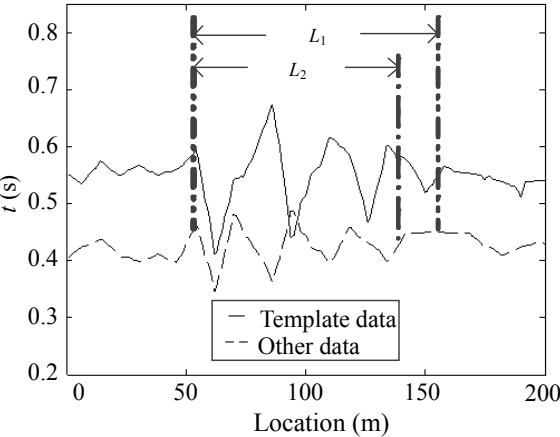

(c)

Fig. 4 Scale of pre-correction signals: (a) picking, (b) shoveling, and (c) running.

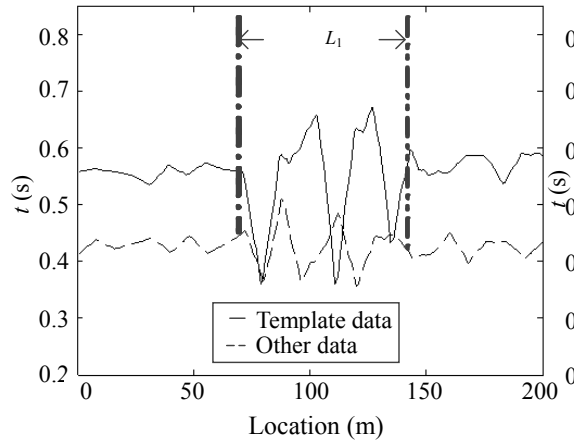

(a)

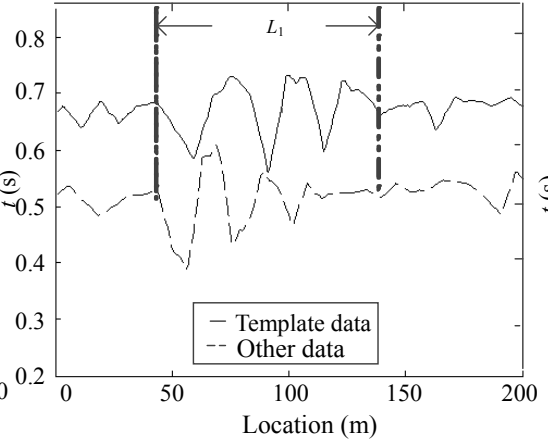

(b)

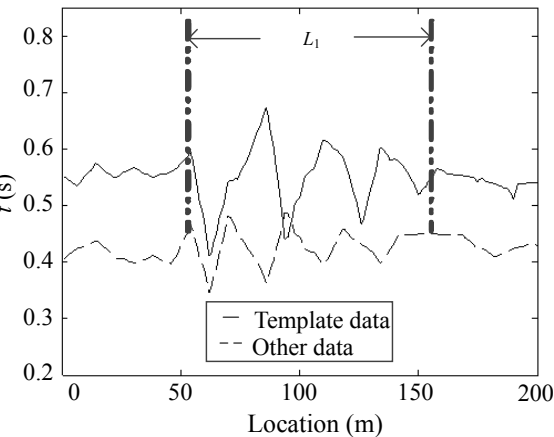

(c)

Fig. 5 Scale of corrected signal: (a) picking, (b) shoveling, and (c) running.

\subsection{BP neural network classification results and analysis}

The Fourier transform is applied for the three kinds of intrusion signals, and the spectrum characteristics are sent into the BP neural network for classification.

The training error is given after 90 times cycle iteration of training samples as shown in Fig. 6. It can be seen from Fig. 6 that the training error of corrected signals is lower than the pre-correction signals, and its descent speed of error is faster than the pre-correction signal when the BP neural network is iteratively trained with the same number of times. It indicates that the network generalization ability is improved because of using the correction method. It is proved that the obtained spectral characteristics in this paper can improve the recognition performance.

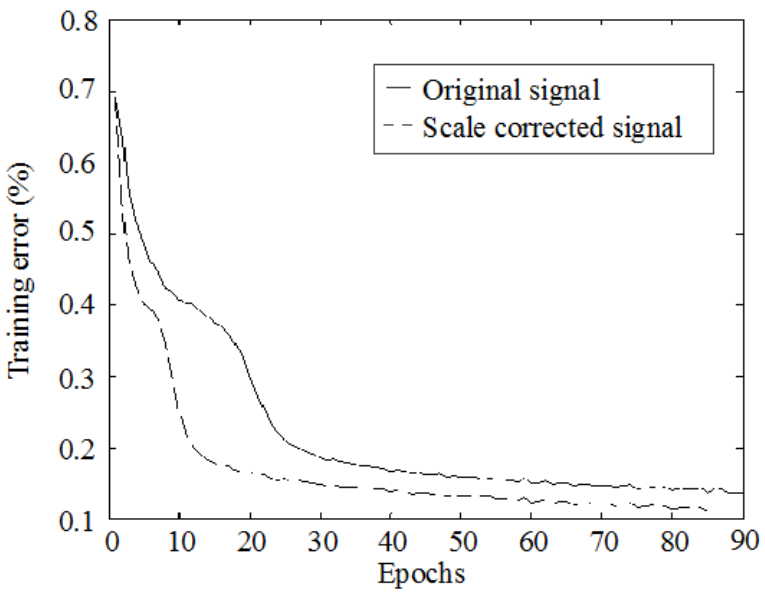

Fig. 6 Training error.

Then, 50 samples of signals in each type are selected for testing. Figure 7 shows the identification results obtained by the three types of signals. As can be seen, the recognition rate of these 
three types of signals is increased by $4 \%, 10 \%$, and $12 \%$, respectively. It is shown that the correction algorithm can effectively improve the recognition accuracy while adapting to the practical application.

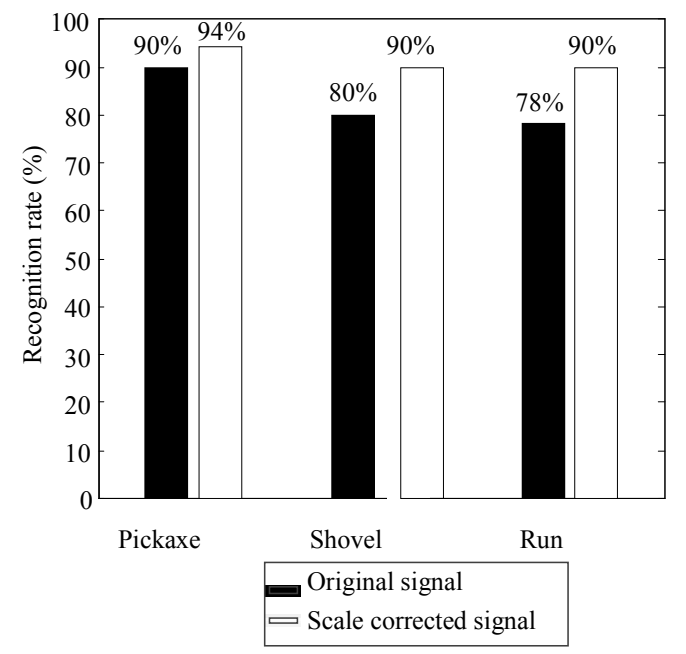

Fig. 7 Signal recognition rate.

\section{Conclusions}

In this paper, we propose a correction method for correcting the scale of intrusion signals in OFPS. In this method, the same kind of signal is adjusted to the same scale by using the MT, and the characteristics with the consistent scale are put into the BP neural network for classification and recognition. The experimental results show that the application of the proposed method can solve the problem of scale transformation, and the recognition rate of the optical fiber intrusion signal is increased by $4 \%, 10 \%$, and $12 \%$, respectively. This scale correction method may also be applied for the further research on the detection and identification of the optical fiber intrusion signal to improve the performance.

\section{Acknowledgement}

This work was supported by the National Natural Science Foundation of China (Grant Nos. 61571014 and 61601006); Beijing Nature Science Foundation (Grant No. 4172017); General Project of Science and Technology Program of Beijing Education Commission (Grant No.
KM201610009004).

Open Access This article is distributed under the terms of the Creative Commons Attribution 4.0 International License (http://creativecommons.org/ licenses/by/4.0/), which permits unrestricted use, distribution, and reproduction in any medium, provided you give appropriate credit to the original author(s) and the source, provide a link to the Creative Commons license, and indicate if changes were made.

\section{References}

[1] Z. Z. Qiu, T. Zheng, H. Q. Qu, and L. P. Pang, "A new method based on CFAR and DE for OFPS," Photonic Sensors, 2016, 6(3): 261-267.

[2] W. Liang, L. L. Lu, and L. B. Zhang, "Coupling relations and early-warning for 'equipment chain' in long-distance pipeline," Mechanical Systems and Signal Processing, 2013, 41(1-2): 335-347.

[3] F. K. Bi, C. Feng, H. Q. Qu, and T. Zheng, "Harmful intrusion detection algorithm of optical fiber pre-warning system based on correlation of orthogonal polarization signals," Photonic Sensors, 2017, 7(3): 226-233.

[4] K. Liu, T. J. Chai, T. G. Liu, J. F. Jiang, Q. N. Chen, L. Pan, et al., "Multi-area optical perimeter security system with quick invasion judgement algorithm," Journal of Optoelectronics Laser, 2015, 26(2): 288-294.

[5] L. J. Hang, C. F. He, B. Wu, D. S. Cai, and Y. R. Song, "Research on novel distributed optical fiber pipeline leakage detection technology and location method," Acta Optica Sinica, 2008, 28(1): 123-127.

[6] C. H. Zhu, Y. Z. Qu, and J. P. Wang, "The vibration signal recognition of optical fiber perimeter based on time-frequency features," Opto-Electronic Engineering, 2014, 41(1): 16-22.

[7] L. Wang, Y. B. Guo, T. G. Sun, J. Y. Huo, and L. Zhang, "Signal recognition of the optical fiber vibration sensor based on two-level feature extraction," in Proceeding of IEEE 8th International Congress on Image and Signal, Shenyang, China, 2015, pp: 1484-1488.

[8] S. S. Mahmoud, Y. Visagathilagar, and J. Katsifolis, "Real-time distributed fiber optic sensor for security systems: performance, event classification and nuisance mitigation," Photonic Sensors, 2012, 2(3): 225-236.

[9] Z. Y. Wang, Z. Q. Pan, Q. Ye, H. W. Cai, R. H. Qu, and Z. J. Fang, "Fast pattern recognition based on frequency spectrum analysis used for intrusion alarming in optic fiber fence," Chinese Journal of 
Lasers, 2015, 42(4): 1-6.

[10] H. F. Li, X. D. Yin, J. Z. Liu, C. Z. Zhang, and Y. Chen, "Intrusion signal recognition basing on optical fiber Bragg grating vibration sensor," Optical Communication Technology, 2012, 2: 12-14.

[11] H. J. Wu, S. K. Xiao, X. Y. Li, Z. N. Wang, J. W. Xu, and Y. J. Rao, "Separation and determination of the disturbing signals in phase-sensitive optical time domain reflectometry ( $\Phi$-OTDR)," Journal of Lightwave Technology, 2015, 33(15): 3156-3162.

[12] R. Sun and W. J. Zeng, "Secure and robust image hashing via compressive sensing," Multedia Tools and Applications, 2014, 70(3): 1651-1665.

[13] B. Xiao, J. F. Ma, and J. T. Cui, "Combined blur, translation, scale and rotation invariant image recognition by Radon and pseudo-Fourier-Mellin transforms," Patern Recogntion, 2012, 45(1):
314-321.

[14] X. C. Dai and Q. Xie, "Research on image matching algorithm based on Fourier-Mellin transform," Infrared Technology, 2016, 38(10): 860-863.

[15] S. W. Zhang, S. C. Zhen, X. L. Zhao, and S. T. Zhao, "Recognition method of radar target using range profile," Systems Engineering and Electronics, 2001, 23(11): 48-51.

[16] J. H. Cheng, G. Gao, W. X. Ding, X. S. Ku, and J. X. Sun, "An improved scheme for parameter estimation of $\mathrm{G}^{\circ}$ distribution model in high-resolution SAR images," Progress in Electromagnetics Research, 2013, 134: 23-46.

[17] J. Yang, T. K. Sarkar, and P. Antonik, "Applying the Fourier-modified Mellin transform (FMMT) to Doppler-istorted waveforms," Digital Singal Processing, 2007, 17(6): 1030-1039. 